\title{
Thiol versus Thiolate Ligation on Cadmium Selenide Quantum Belts
}

\author{
Yuewei Yao and William E. Buhro* \\ Department of Chemistry and Institute of Materials Science and Engineering, \\ Washington University, St. Louis, Missouri 63130-4899. \\ Corresponding Author: buhro@wustl.edu
}

\section{Table of Contents:}

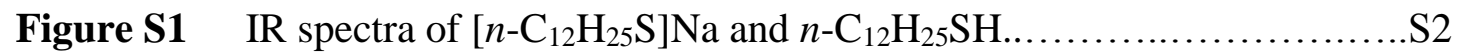

Figure S2 XRD patterns of variously ligated CdSe QBs........................S3

Figure S3 TEM images of variously ligated CdSe QBs..........................S4

Figure S4 Absorption spectra of $\left\{\mathrm{CdSe}\left[n-\mathrm{C}_{12} \mathrm{H}_{25} \mathrm{~S}\right]_{0.23}[\mathrm{Na}]_{0.23}\right\}$ QBs and $\{\mathrm{CdSe}[n-$ dodecanethiol $\left.]_{0.63}\right\}$ QBs obtained by ligand exchange..................S5

Figure S5 Photoluminescence spectra of variously ligated CdSe QBs..............S6

Figure S6 Assignment of the IR spectra of variously ligated CdSe QBs...............S7

Table S1 Combustion-based Elemental Analysis Data, Calculation of Empirical

Formula, and Error Analysis.................................S8 


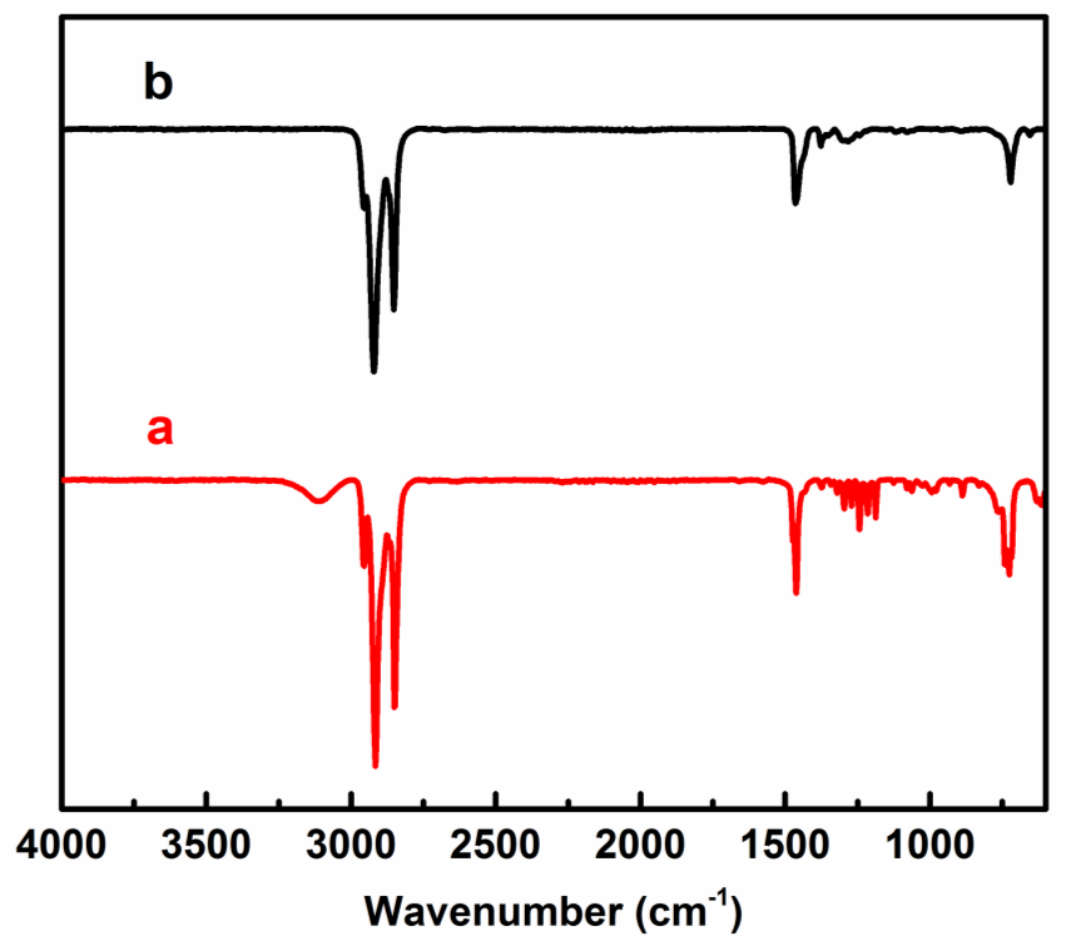

Figure S1. IR spectra of (a) sodium $n$-dodecanethiolate $\left(\left[n-\mathrm{C}_{12} \mathrm{H}_{25} \mathrm{~S}\right] \mathrm{Na}\right)$ and (b) $n$ dodecanethiol $\left(n-\mathrm{C}_{12} \mathrm{H}_{25} \mathrm{SH}\right)$. The S-H stretch at $2579 \mathrm{~cm}^{-1}$ for $n-\mathrm{C}_{12} \mathrm{H}_{25} \mathrm{SH}$ is not detected in the IR spectrum. 


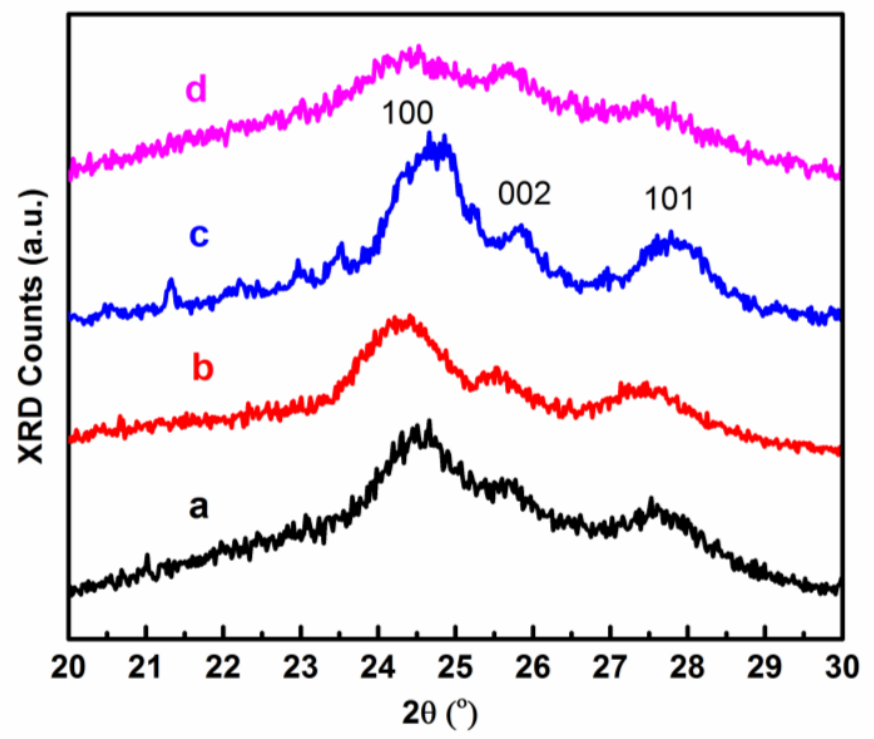

Figure S2. XRD patterns of (a) $\left\{\mathrm{CdSe}\left[n-\mathrm{C}_{12} \mathrm{H}_{25} \mathrm{~S}\right]_{0.23}[\mathrm{Na}]_{0.23}\right\}$ QBs, (b) $\left\{\mathrm{CdSe}\left[\mathrm{Cd}(n \text {-dodecanethiolate })_{2}\right]_{0.22}\right\}$ QBs prepared from $\left\{\mathrm{CdSe}\left[\mathrm{Cd}(\text { oleate })_{2}\right]_{0.19}\right\}$ QBs, (c) $\left\{\mathrm{CdSe}\left[\mathrm{Cd}(n \text {-dodecanethiolate })_{2}\right]_{0.22}\right\}$ QBs prepared from $\{\mathrm{CdSe}[n$ octylamine $\left.]_{0.53}\right\}$ QBs, and (d) $\left\{\mathrm{CdSe}[n \text {-dodecanethiol }]_{0.26}\right\}$ QBs. 


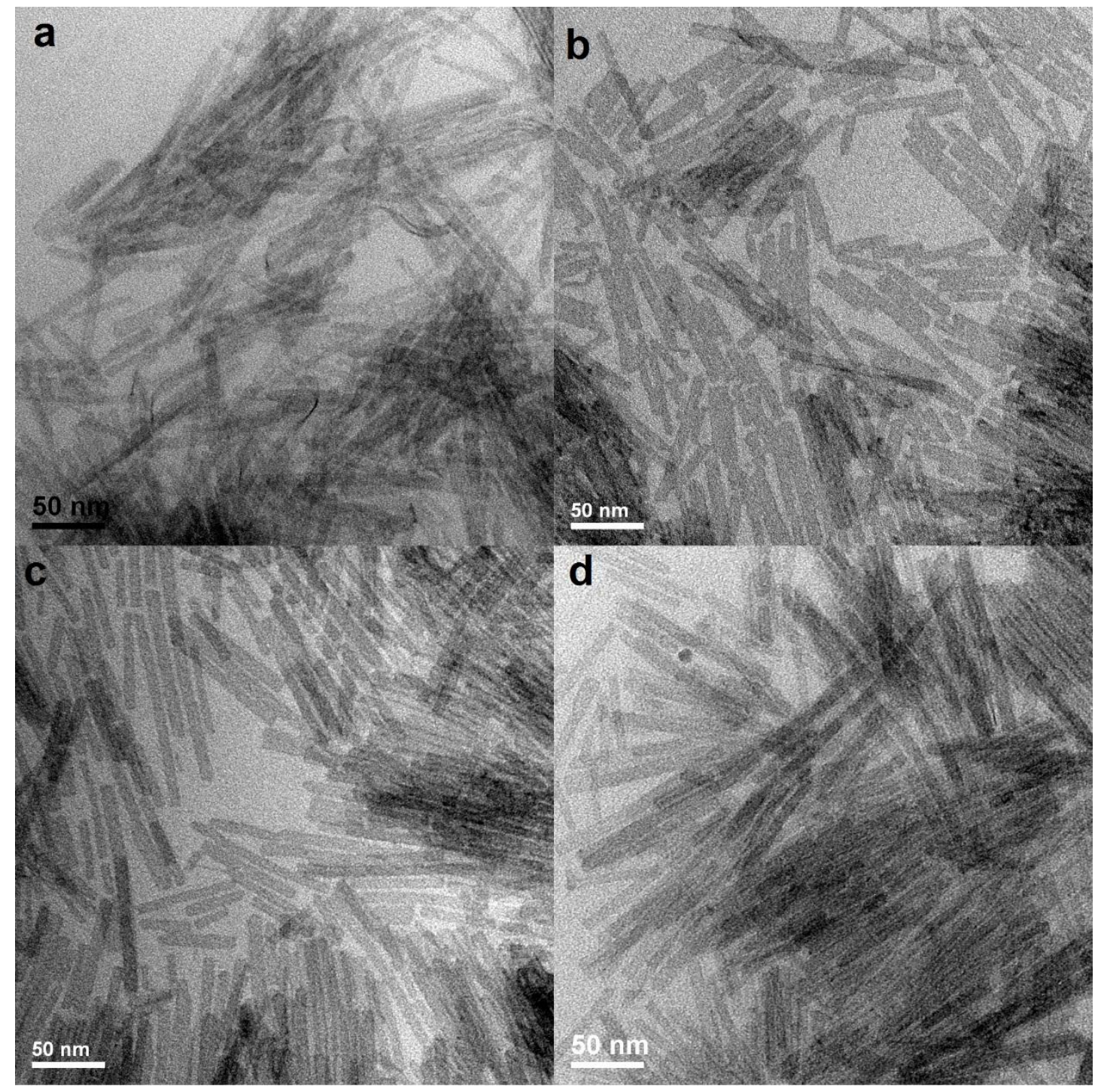

Figure S3. TEM images of (a) $\left\{\mathrm{CdSe}\left[n-\mathrm{C}_{12} \mathrm{H}_{25} \mathrm{~S}\right]_{0.23}[\mathrm{Na}]_{0.23}\right\}$ QBs, (b) $\{\mathrm{CdSe}[\mathrm{Cd}(n-$ dodecanethiolate $\left.\left.)_{2}\right]_{0.22}\right\}$ QBs prepared from $\left\{\mathrm{CdSe}\left[\mathrm{Cd}(\text { oleate })_{2}\right]_{0.19}\right\}$ QBs, (c) $\left\{\mathrm{CdSe}\left[\mathrm{Cd}(n \text {-dodecanethiolate })_{2}\right]_{0.22}\right\}$ QBs prepared from $\left\{\mathrm{CdSe}[n \text {-octylamine }]_{0.53}\right\}$ QBs, and (d) $\left\{\mathrm{CdSe}[n \text {-dodecanethiol }]_{0.26}\right\}$ QBs. 


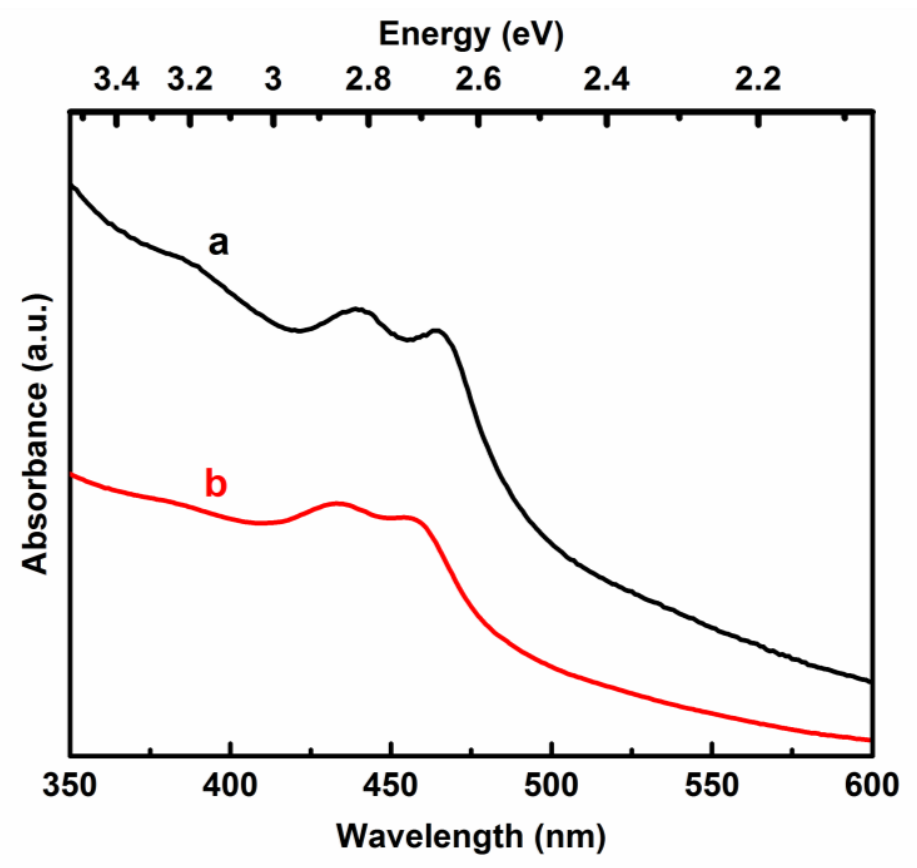

Figure S4. Absorption spectra of (a) $\left\{\mathrm{CdSe}\left[n-\mathrm{C}_{12} \mathrm{H}_{25} \mathrm{~S}\right]_{0.23}[\mathrm{Na}]_{0.23}\right\}$ QBs, and (b) $\left\{\mathrm{CdSe}[n \text {-dodecanethiol }]_{0.63}\right\}$ QBs obtained by ligand exchange of $\{\mathrm{CdSe}[n$ $\left.\mathrm{C}_{12} \mathrm{H}_{25} \mathrm{~S}\right]_{0.23}[\mathrm{Na}]_{0.23}$ QBs with $n$-octylamine. 


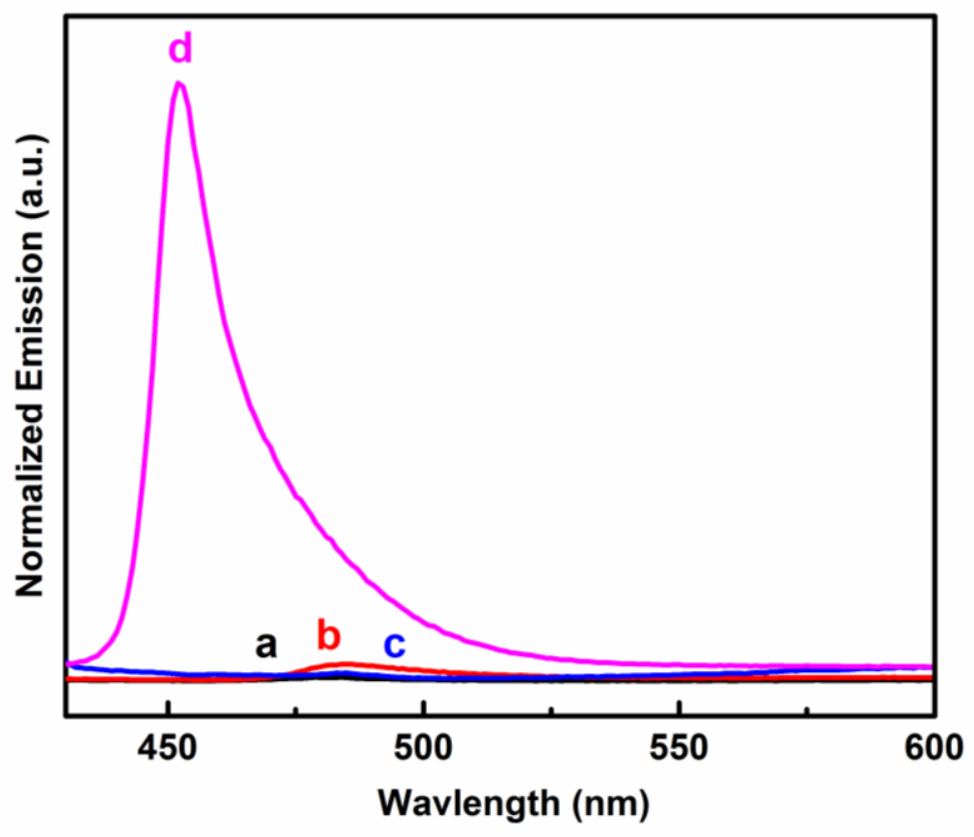

Figure S5. Photoluminescence spectra of (a) $\left\{\mathrm{CdSe}\left[n-\mathrm{C}_{12} \mathrm{H}_{25} \mathrm{~S}\right]_{0.23}[\mathrm{Na}]_{0.23}\right\}$ QBs, (b) $\left\{\mathrm{CdSe}\left[\mathrm{Cd}(n \text {-dodecanethiolate })_{2}\right]_{0.22}\right\} \mathrm{QBs},(\mathrm{c})\left\{\mathrm{CdSe}[n \text {-dodecanethiol }]_{0.26}\right\}$ QBs, and (d) $\left\{\mathrm{CdSe}[n \text {-octylamine }]_{0.53}\right\}$ QBs obtained by ligand exchange of $\{\mathrm{CdSe}[\mathrm{Cd}(n-$ dodecanethiolate) $\left.\left.{ }_{2}\right]_{0.22}\right\}$ QBs with $n$-octylamine. 


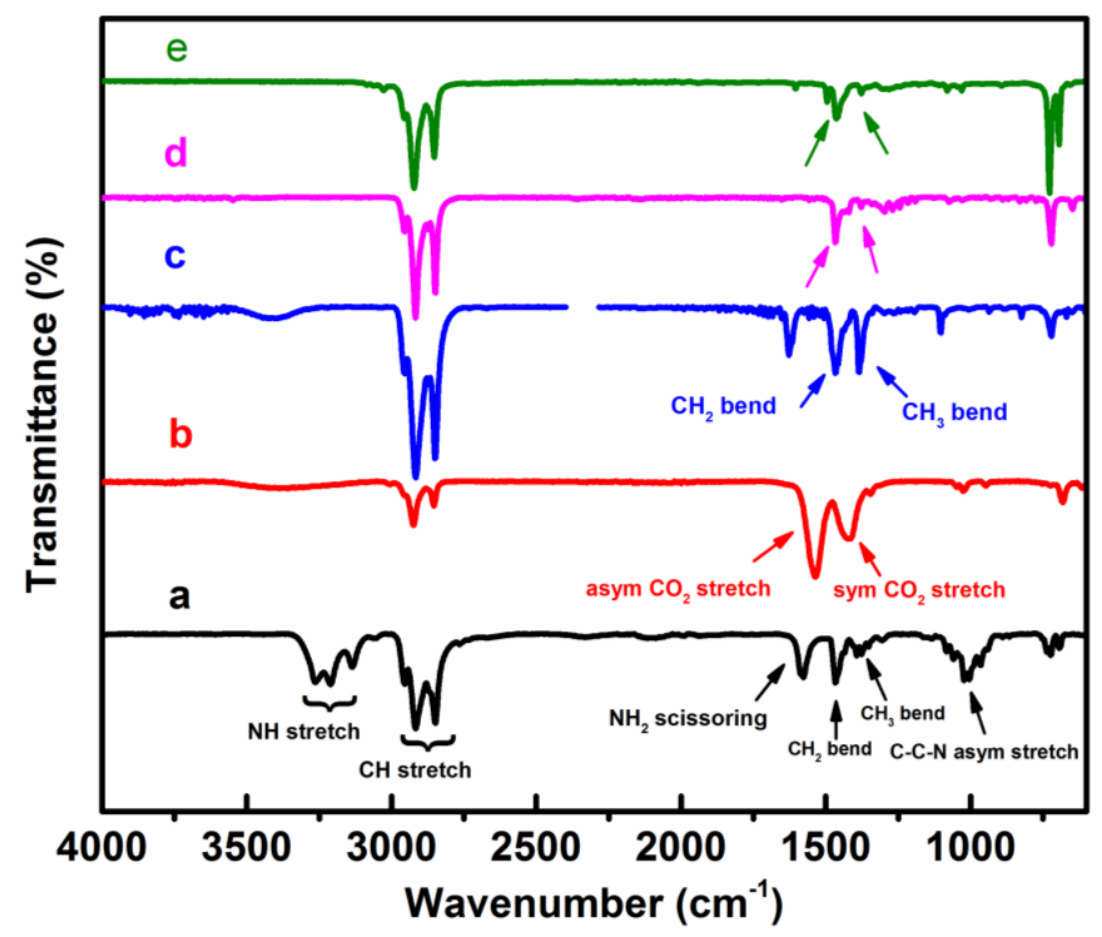

Figure S6. IR spectra of (a) $\left\{\mathrm{CdSe}[n \text {-octylamine }]_{0.53}\right\}$ QBs; NH stretching range $3300-3100 \mathrm{~cm}^{-1}$; $\mathrm{CH}$ stretching range $3000-2800 \mathrm{~cm}^{-1}$; $\mathrm{NH}_{2}$ scissoring at $1578 \mathrm{~cm}^{-1}$; $\mathrm{CH}_{2}$ bend at $1459 \mathrm{~cm}^{-1} ; \mathrm{CH}_{3}$ bend at $1379 \mathrm{~cm}^{-1}$; C-C-N asymmetric stretch at 1011 $\mathrm{cm}^{-1}$; (b) $\left\{\mathrm{CdSe}\left[\mathrm{Cd}(\text { oleate })_{2}\right]_{0.19}\right\}$ QBs; asymmetric $\mathrm{CO}_{2}$ stretch at $1534 \mathrm{~cm}^{-1}$ and symmetric $\mathrm{CO}_{2}$ stretch at $1423 \mathrm{~cm}^{-1}$; (c) $\left\{\mathrm{CdSe}\left[n-\mathrm{C}_{12} \mathrm{H}_{25} \mathrm{~S}\right]_{0.23}[\mathrm{Na}]_{0.23}\right\} \mathrm{QBs} ; \mathrm{CH}_{2}$ bend at $1467 \mathrm{~cm}^{-1} ; \mathrm{CH}_{3}$ bend at $1384 \mathrm{~cm}^{-1} ;$ (d) $\left\{\mathrm{CdSe}\left[\mathrm{Cd}(n \text {-dodecanethiolate })_{2}\right]_{0.22}\right\}$ QBs; $\mathrm{CH}_{2}$ bend at $1467 \mathrm{~cm}^{-1} ; \mathrm{CH}_{3}$ bend at $1379 \mathrm{~cm}^{-1}$; (e) $\left\{\mathrm{CdSe}[n \text {-dodecanethiol }]_{0.63}\right\}$ QBs, $\mathrm{CH}_{2}$ bend at $1459 \mathrm{~cm}^{-1} ; \mathrm{CH}_{3}$ bend at $1374 \mathrm{~cm}^{-1}$. 
Table S1. Combustion-based Elemental Analysis Data.

\begin{tabular}{ccccc}
$\mathrm{CdSe}\left[n-\mathrm{C}_{12} \mathrm{H}_{25} \mathrm{~S}\right]_{0.23 \pm 0.02}[\mathrm{Na}]_{0.23 \pm 0.02}$ & $\mathrm{C}(\%)$ & $\mathrm{H}(\%)$ & $\mathrm{N}(\%)$ & $\mathrm{S}(\%)$ \\
Calcd & 13.64 & 2.39 & $\mathrm{NA}$ & 3.03 \\
Found & 14.01 & 2.19 & $<0.5$ & 3.17 \\
\hline $\mathrm{CdSe}\left[\mathrm{Cd}(n \text {-dodecanethiolate })_{2}\right]_{0.22 \pm 0.02}$ & $\mathrm{C}(\%)$ & $\mathrm{H}(\%)$ & $\mathrm{N}(\%)$ & $\mathrm{S}(\%)$ \\
\hline Calcd & 20.81 & 3.64 & $\mathrm{NA}$ & 4.63 \\
Found & 21.78 & 4.39 & $<0.5$ & 4.10 \\
$\mathrm{CdSe}[n \text {-dodecanethiol }]_{0.63 \pm 0.02}$ & $\mathrm{C}(\%)$ & $\mathrm{H}(\%)$ & $\mathrm{N}(\%)$ & $\mathrm{S}(\%)$ \\
Calcd & 38.53 & 4.03 & $\mathrm{NA}$ & 4.93 \\
Found & 38.46 & 4.39 & $<0.5$ & 5.38 \\
Found & 39.96 & 4.45 & $<0.5$ & 5.44 \\
$\mathrm{CdSe}[n \text {-dodecanethiol }]_{0.26 \pm 0.02}$ & $\mathrm{C}(\%)$ & $\mathrm{H} \mathrm{( \% )}$ & $\mathrm{N}(\%)$ & $\mathrm{S}(\%)$ \\
Calcd & 15.36 & 2.79 & $\mathrm{NA}$ & 3.42 \\
Found & 13.46 & 2.18 & $<0.5$ & 2.99
\end{tabular}

Example calculation of empirical formula (and error) from elemental-analysis data: determining the empirical formula of $\mathrm{CdSe}[n \text {-dodecanethiol }]_{0.63 \pm 0.02}$

$\mathrm{N}_{\text {mol }} \mathrm{S}: \quad 5.38 / 32.06=0.168 \mathrm{~mol}$

$\mathrm{N}_{\mathrm{mol}} \mathrm{H}: \quad 4.39 /(26 * 1.008)=0.168 \mathrm{~mol}$

$\mathrm{N}_{\text {mol }}$ CdSe: $\quad(100 \mathrm{~g}-4.39 \mathrm{~g}-5.38 \mathrm{~g}-38.46 \mathrm{~g}) / 191.4 \mathrm{~g} / \mathrm{mol}=0.270 \mathrm{~mol}$

If we assume the empirical formula is $\mathrm{CdSe}[\mathrm{n} \text {-dodecanethiol }]_{\mathrm{x}}$

$0.270 / 0.168=1 / x, \quad x=0.62$

$\mathrm{N}_{\text {mol }} \mathrm{S}: \quad 5.44 / 32.06=0.170 \mathrm{~mol}$

$\mathrm{N}_{\mathrm{mol}} \mathrm{H}: \quad 4.45 / 26 * 1.008=0.170 \mathrm{~mol}$

$\mathrm{N}_{\mathrm{mol}} \mathrm{CdSe}: \quad(100 \mathrm{~g}-4.45 \mathrm{~g}-5.44 \mathrm{~g}-39.97 \mathrm{~g}) / 191.4 \mathrm{~g} / \mathrm{mol}=0.262 \mathrm{~mol}$

If we assume the empirical formula is $\mathrm{CdSe}[\mathrm{n} \text {-dodecanethiol }]_{\mathrm{x}}$

$0.262 / 0.170=1 / x, \quad x=0.65$

$(0.62+0.65) / 2=0.63 \pm 0.02$

Thus the empirical formula is $\mathrm{CdSe}[\mathrm{n} \text {-dodecanethiol }]_{0.63 \pm 0.02}$

Errors in the $\mathrm{Cd} / \mathrm{Se}$ ratios measured by EDS were the standard deviations from multiple measurements of the same specimen. 